\title{
Enhanced Recovery in the ICU after Cardiac Surgery \& New Developments in Cardiopulmonary Resuscitation
}

Editors

DANIEL T. ENGELMAN

CLIFTON W. CALLAWAY

CRITICAL CARE

CLINICS

www.criticalcare.theclinics.com

Consulting Editor

JOHN A. KELLUM

October 2020 • Volume $36 \bullet$ Number 4 